\title{
Multi-Objective Optimal Operation of the Inter-Basin Water Transfer Project Considering the Unknown Shapes of Pareto Fronts
}

\author{
Jianjian $\mathrm{Xu}^{1,2}$ and Dan Bai ${ }^{1, *}$ \\ 1 State Key Laboratory of Eco-Hydraulics in Northwest Arid Region of China, Xi'an University of Technology, \\ Xi'an 710048, China; xujj760517@163.com \\ 2 Shanxi Province Institute of Water Resources and Electric Power Investigation and Design, \\ Xi'an 710001, China \\ * Correspondence: baidan@xaut.edu.cn
}

Received: 17 October 2019; Accepted: 11 December 2019; Published: 14 December 2019

\begin{abstract}
Studies have shown that the performance of multi-objective evolutionary algorithms depends to a large extent on the shape of the Pareto fronts of the problem. Although, most existing algorithms have poor applicability in dealing with this problem, especially in the multi-objective optimization operation of reservoirs with unknown Pareto fronts. Therefore, this paper introduces an evolutionary algorithm with strong versatility and robustness named the Multi-Objective Evolutionary Algorithm with Reference Point Adaptation (AR-MOEA). In this paper, we take two water conservancy hubs (Huangjinxia and Sanhekou) of the Hanjiang to Wei River Water Diversion Project as example, and build a multi-objective operation model including water supply, ecology, and power generation. We use the AR-MOEA, the Non-dominated Sorting Genetic Algorithm II (NSGA-II), the Multi-Objective Evolutionary Algorithm based on Decomposition (MOEA/D) and the Indicator-Based Evolutionary Algorithm (IBEA) to search the optimal solutions, respectively. We analyze the performance of four algorithms and the operation rules in continuous dry years. The results indicate that (1) the AR-MOEA can overcome the difficulty of the shape and distribution of the unknown Pareto fronts in the multi-objective model. (2) AR-MOEA can improve the convergence and uniformity of the Pareto solution. (3) If we make full use of the regulation ability of the Sanhekou reservoir in the dry season, the water supply for coping with possible continuous dry years can be guaranteed. The study results contribute to the identification of the relationship among objectives, and is valued for water resources management of the Hanjiang to Wei River Water Diversion Project.
\end{abstract}

Keywords: AR-MOEA; reservoir optimization operation; unknown shapes of Pareto fronts; Hanjiang to Wei River Water Diversion Project

\section{Introduction}

Water resources play an increasingly indispensable role in regional development with the development of social economy and the acceleration of urbanization [1]. Climate change and human activities have intensified the spatial and temporal distribution of water resources in some regions [2,3], which further causes resource-based and water-based water shortages. As an important engineering measure to change the spatial and temporal distribution of water resources, the inter-basin water transfer project can effectively alleviate the contradiction between water supply and demand and improve the ecological environment in the water receiving area [4,5], such as the South-to-North Water Transfer Project of China [6]. The inter-basin water transfer project usually includes reservoir-pump-power station groups, water-storage user groups, and long-distance water transport facilities [7]. The optimized operation generally has the characteristics of high dimension, 
multi-objective, and strict constraints. Therefore, as a bridge connecting two or even multiple basins, multi-objective optimization operation of inter-basin water transfer projects has become one of the hot research topics in the current reservoir operation field.

At present, the research on the operation of the inter-basin water transfer projects is mainly focused on the reservoir group operation rules in the water source area and the water receiving area. For instance, Zhou et al. [8] proposes an intelligent water allocation methodology for establishing optimal inter-basin water allocation schemes, and solved the joint operation map of water diversion and water supply of the reservoir and its operation rules. Zeng et al. [9] proposed a new triggering mechanism by establishing an inter-basin water transfer model to search the operating objectives for meeting annual diversion requirements. Guo et al. [10] established a reservoir group optimal operation model based on the $0-1$ planning method, and Progressive Optimization Algorithm (POA) is used to solve the optimal water supply process. Wan et al. [11] built up a tri-level programming model based on the game theory and proposed inter-basin reservoir group optimization operation rules after combining water transfer, water diversion, and water supply. All of the above studies have achieved good results in model construction and rules extraction. However, the advantages of Multi-Objective Evolutionary Algorithm (MOEA) [12] in multi-objective models are not fully utilized. Therefore, combining mature MOEA with multi-objective optimization operation of the inter-basin water transfer project remains challenging.

Compared with the traditional multi-objective method, the Pareto solution set of MOEA includes the optimal solution of all the model objectives. It has the advantages of strong robustness and objectivity, and few human interference factors. Nowadays, many MOEAs have been developed to solve various water resources problems. For instance, Reddy et al. [13] combined the elite mutation strategy with the multi-objective particle swarm optimization algorithm (MOPSO) to solve the multi-objective model of the Indian Bhadra reservoir which considering the irrigation, power generation, and water quality. Yu et al. [14] established a multi-objective ecological reservoir operations model of the Three Gorges Reservoir which based on the indicators of hydrologic alteration (IHA) and the histogram matching approach (HMA), and the model is solved by the Non-dominated Sorting Genetic Algorithm II (NSGA-II). Uen et al. [15] used the (NSGA-II) to optimize the water-food-energy multi-objective problem in a reservoir. Niu et al. [16] presented a parallel multi-objective particle swarm optimization (PMOPSO) to solve the cascade hydropower reservoir operation, where the swarm with large population size is divided into several smaller sub swarms to be simultaneously optimized by different worker threads. Qi et al. [17] developed an enhanced algorithm with adaptive neighborhood size and genetic operator selection based on the MOEA to solve a multi-objective reservoir flood control operation problem. Bai et al. [18] proposed an innovative approach that hybrids the Feasible Search Space (FSS) with the MOPSO algorithm to search the optimal solutions for the multi-objective operation of cascade reservoirs in Yellow river. However, unlike the theoretical test function model, as the number of reservoirs, objectives, and complexity of the problem increases, the degree of solution to the multi-objective optimization model of the actual problem increases, especially in the multi-objective operation model of the reservoirs group.

Ishibuchi et al. [19] presented that the solution performance of MOEA depends largely on the Paetro front shapes of the problem to be solved, and different algorithms have different sensitivity to different shapes. Among them, the regular fronts shape means continuous, smooth, and well distributed, and the irregular shapes refer to discontinuities, inversions, or sharp tails. This study indicates that if the Pareto front shape is known, the evolutionary direction of the algorithm will be more purposeful, and the convergence will be better. In contrast, the efficiency of the solution, the diversity, and uniformity of the Pareto solution set will be reduced. Meanwhile, the uncertainty of the performance of different MOEAs for multi-objective models is greater due to different sensitivities, especially in the multi-objective optimization of the actual engineering problems. Tian et al. [20] proposed a Multi-Objective Evolutionary Algorithm with Reference Point Adaptation (AR-MOEA) in order to solve the above problems. The core idea of AR-MOEA is to add a screening mechanism for 
candidate solutions in the multi-objective evolutionary algorithm based on indicators. Meanwhile, AR-MOEA uses Enhanced Inverted Generational Distance (IGD-NS) as the criterion for algorithm selection to distinguish and eliminate solutions that do not contribute to IGD-NS, and accelerate the evolution of algorithms to Pareto fronts. In the process of calculating the value of IGD-NS by using the reference point, the AR-MOEA adaptively adjusts and updates a set of reference points by uniform sampling and according to the contribution of the solution. And the AR-MOEA can show good robustness and versatility in dealing with unknown Pareto front problems. In summary, this paper will introduce AR-MOEA with higher applicability and versatility to solve the multi-objective reservoir operation model with high dimensionality and an unknown Pareto front surface.

In this study, Hanjiang to Wei River Water Diversion Project of Shaanxi Province is taken as the study case, and a multi-objective optimization model is established considering three important objectives (water supply, ecology, and power generation). The reservoir-power station-pump group is taken as the main content of the operation. Four algorithms, named NSGA-II, the Multi-objective Evolutionary Algorithm based on Decomposition (MOEA/D), the Indicator-Based Evolutionary Algorithm (IBEA), and the AR-MOEA are used to solve the multi-objective model, respectively. The study compares the convergence of the four algorithms and the uniformity of the solution, and formulates the project operation strategy for the continuous dry years. This research can provide a new idea for solving the multi-objective optimization operation model of the reservoir, and provide a certain reference value for the development of the operational rules of the Hanjiang to Wei River Water Diversion Project.

\section{Research Area and Data}

The Hanjiang to Wei River Water Diversion Project, located in Shaanxi Province of China, transfers water from Han River where the water resources are relatively rich to the Guanzhong area of Weihe River where the water shortage is severe. The project will not only alleviate the contradiction between water supply and demand in the Guanzhong area of the Weihe River Basin, but also will improve the ecological environment of the Weihe River Basin. The whole project includes a water transfer project and water distribution project. Among them, the water transfer project is mainly composed of three parts: two water source area projects (the Huangjinxia and the Sanhekou water conservancy hubs) and the Qinling Water Transmission Tunnel (water transport medium). Both the Huangjinxia and the Sanhekou water conservancy hubs contains the reservoir, hydropower station, and pumping station. The schematic of the Hanjiang to Wei River Water Diversion Project is shown in Figure 1, and the values of the characteristic parameters for the project are given in Table 1.

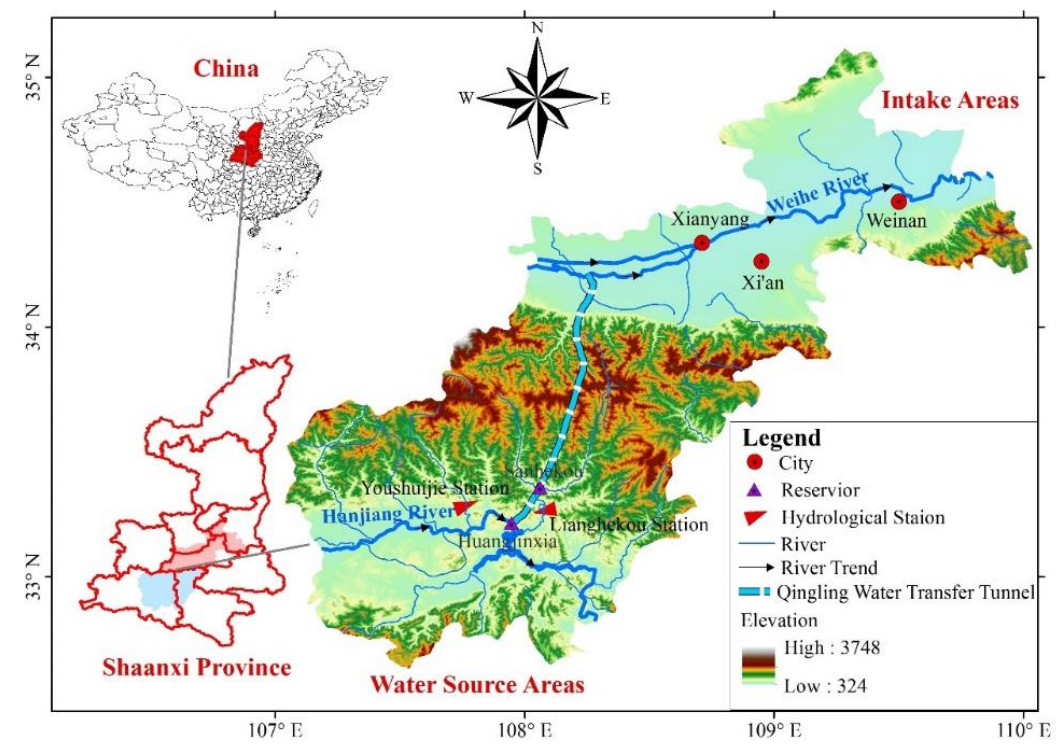

Figure 1. Schematic of the Hanjiang to Wei River Water Diversion Project. 
Table 1. Characteristic parameters for Hanjiang to Wei River Water Diversion Project.

\begin{tabular}{lcc}
\hline Characteristic Parameters & Huangjinxia & Sanhekou \\
\hline Regulation ability & Daily & Multi-year \\
Total storage $\left(10^{8} \mathrm{~m}^{3}\right)$ & 2.29 & 7.1 \\
Regulating storage $\left(10^{8} \mathrm{~m}^{3}\right)$ & 0.92 & 6.49 \\
Normal water level $(\mathrm{m})$ & 450 & 643 \\
Water level for flood control (m) & 482 & 644 \\
Dead water level (m) & 440 & 558 \\
Installed capacity (MW) & 135 & 64 \\
Firm capacity (MW) & 8.6 & - \\
Pump station installed capacity $(\mathrm{MW})$ & 135 & 24 \\
Pumping station pumping flow $\left(\mathrm{m}^{3} / \mathrm{s}\right)$ & 70 & 18 \\
Ecological flow $\left(\mathrm{m}^{3} / \mathrm{s}\right)$ & 25 & 2.71 \\
\hline
\end{tabular}

The designed water transfer scale of the project is 1.5 billion $\mathrm{m}^{3}$ average annually under the premise of ensuring the water volume of the national South-to-North Water Transfer Project. The overall operating rules of the project is that the Huangjinxia pumping station lifts water from the Huangjinxia reservoir to the control gate of the Qinling Water Transmission Tunnel first. If the water supply of the Huangjinxia reservoir can meet the water demand of the Guanzhong area, the Sanhekou reservoir does not participate in water supply, and the excess water volume can be stored in the Sanhekou reservoir through the Sanhekou pumping station. On the contrary, the Sanhekou reservoir begins to supplement the water supply. Among them, the Huangjinxia reservoir needs energy consumption when it supplies water, and the Sanhekou reservoir is self-flowing water supply. Meanwhile, the considerations of the operation of the Hanjiang to Wei River Water Diversion Project on this paper are focused on the following three points:

(1) The operation of the project needs to weigh the interests of many aspects.

The project involves the river ecology (ecological benefits), the water distribution of the Han River (social benefits), and the external water supply of the Guanzhong area (social, economic, and ecological benefits), operating costs of the project's own power station and pumping station (economic benefits). Therefore, the optimization operation of the entire project is necessarily a multi-objective problem that includes ecological, economic, and social benefits.

(2) The water quantity and the reservoir regulation ability do not match.

The regulation ability of the Huangjinxia reservoir is only daily regulation, although the Han River has abundant water resources. On the contrary, the regulation ability of the Sanhekou reservoir is multi-year regulation, although the runoff of the Ziwu River Basin is only 1/8 of the Han River. Therefore, it is very important to determine the supply and storage order of reservoirs in order to give full play to the water volume advantage of the Han River and the regulation performance of the Sanhekou Reservoir.

(3) Complicated constraints of project.

The constraints mainly include the pumping capacity of the pumping station, the water delivery capacity of the tunnel, the water level of reservoir, and the adjustable water volume of the Han River, etc.

\section{Multi-Objective Optimization Model}

With rapid economic development, the role of reservoirs has become substantially important to meet energy and water requirements [21,22]. Serving as important regulation hubs of the Hanjiang to Wei River Water Diversion Project, the Huangjinxia reservoir and the Sanhekou reservoir have undertaken comprehensive resource utilization tasks. Combined with the actual situation of the project, this paper establishes a multi-objective operation model that considers water supply, ecology, and power generation. The operation period is the monthly scale, and the time-delay effect of flow evolution is not considered in the model. Meanwhile, the operation model is limited to the reservoir between the water sources for there is no corresponding regulating reservoir for the water receiving area. 


\subsection{Objective Functions}

\subsubsection{Water Supply}

Water supply is one of the most important objectives in this study. To meet water demands, it is necessary to ensure that the water supply process is as consistent as possible with the water demand process. Therefore, the water supply objective can be expressed by Water Shortage Index of Intake Area (WSI_IA) minimum, shown as follows.

$$
\begin{gathered}
W S I \_I A=\operatorname{Min} \sum_{i=1}^{T}\left(\frac{q_{-} t(i)-q_{-} d(i)}{q_{-} d(i)}\right)^{2} \cdot 100 \%, \\
q_{t(i)}=q_{H}^{p}(i)+q_{s}(i)-q_{H 2 S}^{p}(i),
\end{gathered}
$$

where $q_{-} t(i)$ and $q_{-} d(i)$ are the water delivery flow of the Qinling Water Transmission Tunnel and the water demand flow in the water receiving area at time $i$, respectively. $q_{H}^{p}(i)$ is the pumping water flow the Huangjinxia pumping station at time $i . q_{s}(i)$ is the water supply flow of the Sanhekou Reservoir at time $i . q_{H 2 S}^{p}(i)$ is the water supply flow to the Sanhekou reservoir from the Huangjinxia reservoir at time $i . T$ is the number of operation periods.

\subsubsection{Ecological Flow Guarantee}

The ecological object selected is the downstream of the Huangjinxia reservoir and Sanhekou reservoir in the water source area. The minimum duration Guarantee Rate of Ecological Flow (GR_E) is the maximum, shown as follows.

$$
\begin{gathered}
G R \_E=\operatorname{Max}\left\{\operatorname{Min}\left(G R \_E \_H, G R \_E \_S\right)\right\}, \\
G R \_E \_H=\frac{T_{H}}{T+1} \cdot 100 \%, \\
G R \_E \_S=\frac{T S}{T+1} \cdot 100 \%,
\end{gathered}
$$

where GR_E_H and GR_E_S are the diachronic ecological guarantee rates of the downstream of the Huangjinxia reservoir and the downstream of the Sanhekou reservoir, respectively. $T \_H$ and $T \_S$ are the number of periods that meet ecological flow of the downstream of the Huangjinxia reservoir and Sanhekou reservoir, respectively.

\subsubsection{Net Power Generation}

The Hanjiang to Wei River Water Diversion Project includes two power stations and two pumping station groups. Among them, the Sanhekou pumping station contains two-way units, which act as generators when supplying water, and act as pumping stations when replenishing water to the Sanhekou reservoir. The net power of the entire system $\left(P \_n e t\right)$ is one of the important optimization objectives of the project's own management, shown as follows.

$$
\begin{gathered}
P \_n e t=\operatorname{Max} \sum_{i=1}^{T} \sum_{n=1}^{N}\left[E_{P}^{n}(i)-E_{C}^{n}(i)\right], \\
E_{P}^{n}(i)=k_{P}^{n} \cdot Q_{o}(i) \cdot H_{P}^{n}(i) \cdot \Delta t, \\
E_{C}^{n}(i)=\frac{g \cdot q_{C}^{n}(i) \cdot H_{C}^{n}(i)}{\eta_{C}^{n}} \cdot \Delta t,
\end{gathered}
$$

where $E_{P}^{n}(i)$ and $E_{C}^{n}(i)$ are the power generation and energy consumption of the power station and pump station at time $i$, respectively. $k_{P}^{n}(i)$ and $\eta_{C}^{n}(i)$ are the output coefficient of the power station and the energy conversion efficiency of the pump station at time $i$, respectively. $Q_{o}(i)$ and $q_{C}^{n}(i)$ are 
the power generation flow of the power station and the pumping flow of the pump station at time $i$, respectively. $H_{P}^{n}(i)$ and $H_{C}^{n}(i)$ are the working heads of the power generating head and pumping station of the power station at time $i$, respectively. $\Delta t$ is the time interval. When $n=1$, it represents the Huangjinxia water conservancy hub and when $n=2$, it represents the Sanhekou water conservancy hub.

\subsection{Constraints}

(1) Water balance

$$
V(n, i+1)=V(n, i)+[Q I(n, i)-Q O(n, i)-Q S(n, i)] \cdot \Delta t,
$$

where $V(n, i+1)$ and $V(n, t)$ are the initial storages of the $n$th reservoir at times $i+1$ and $i$, respectively. $Q I(n, i), Q O(n, i)$, and $Q S(n, i)$ are the inflow, outflow, and the water-transferred flow of the nth reservoir at time $i$, respectively. $\Delta t$ is the time interval.

(2) Water level

$$
Z_{\min }(S H K, i) \leq \mathrm{Z}(S H K, i) \leq Z_{\max }(S H K, i),
$$

where $Z(S H K, i)$ is the water level of the Sanhekou reservoir at time $i . Z_{\min }(S H K, i)$ and $Z_{\max }(S H K, i)$ are the minimum and the maximum water levels of the Sanhekou reservoir at time $i$, respectively.

(3) Output of power station

$$
N_{\min }(n, i) \leq N(n, i) \leq N_{\max }(n, i),
$$

where $N(n, i)$ is the output of $n$th power station at time $i . N_{\min }(n, i)$ and $N_{\max }(n, i)$ are the minimum and the maximum output of $n$th power station at time $i$, respectively.

(4) Power of pump station

$$
P(n, i) \leq P_{\max }(n, i),
$$

where $P(n, i)$ is the power consumption of the $n$th pump station at time $i . P_{\max }(n, i)$ is the maximum power consumption of the $n$th pump station at time $i$.

(5) Flow

$$
\begin{aligned}
& Q P(n, i) \leq Q P_{\text {max }}(n, i), \\
& Q P(n, i) \leq Q P_{\text {max }}(n, i),
\end{aligned}
$$

where $Q P(n, i)$ and $Q P_{\max }(n, i)$ are the outflow and maximum outflow of $n$th power station at time $i$. $Q T(Q L, i)$ and $Q T_{\max }(Q L, i)$ are the transfer flow and the maximum transfer flow of Qinling tunnel at time $i$, respectively.

\section{Search Methods}

In recent years, many researchers have developed a number of multi-objective evolutionary algorithms that are used to deal with many types of multi-objective problems and obtain the most complete Pareto solution set. According to different core algorithm designs, these algorithms mainly include three types:

(1) Multi-objective evolutionary algorithm based on Pareto's solution dominance level. According to the dominance of the solution set, the corresponding population strategy is selected to perform the population selection operation. Representative algorithms include NSGA-II, SPEA2, and PESA-II, etc. [22-26].

(2) Multi-objective evolutionary algorithm based on decomposition. Its idea is to decompose the multi-objective problem into several single-objective problems or simpler multi-objective problems. Representative algorithms include MOEA/D, RVEA, etc. [27-29].

(3) Multi-objective evolutionary algorithm based on performance indicators. Its idea is selecting individuals with high evaluation level and obtained Pareto solution set by setting evaluation indicators as the environment selection strategy in the process of population evolution. Representative algorithms include GDE-MOEA, IBEA, etc. [30,31]. 
In this paper, the AR-MOEA is adopted to solve the multi-objective model, which belongs to the third multi-objective evolutionary algorithm. To further analyze the performance of the algorithm, this paper selected NSGA-II, MOEA/D, IBEA, and AR-MOEA [12], four methods to solve the multi-objective model.

\subsection{AR-MOEA}

The advantages of AR-MOEA are mainly reflected in performance indicators IGD-NS, reference point adaptive strategy, and environment selection strategy. The flowchart of AR-MOEA is shown in Figure 2.

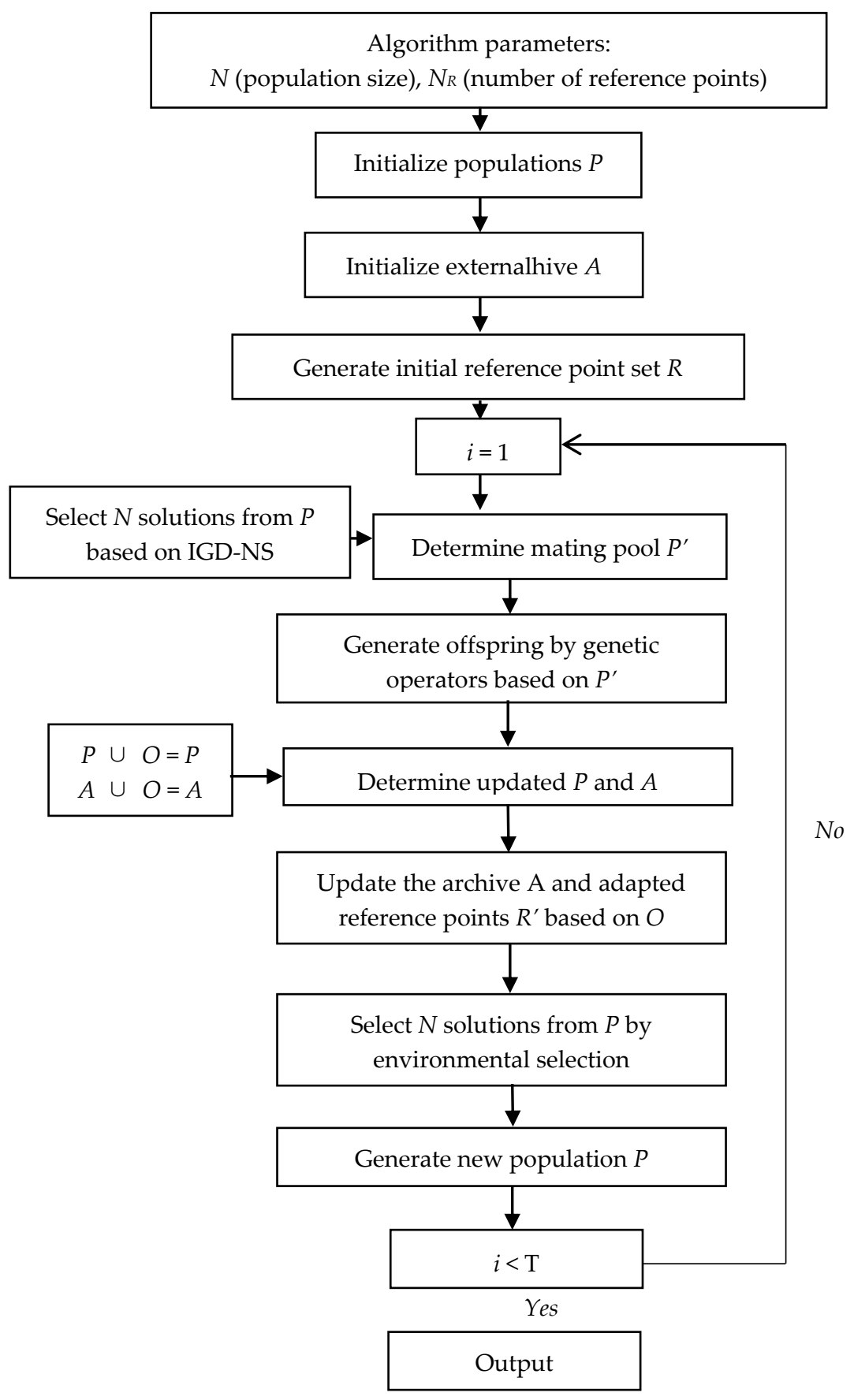

Figure 2. Flowchart of the Multi-Objective Evolutionary Algorithm with Reference Point Adaptation (AR-MOEA). 
(1) Performance index IGD-NS

Every indicator has its own advantages and disadvantages, such as, Inverted Generational Distance (IGD) and Generational Distance (GD) require Pareto front surfaces to be calculated. Hyper-volume (HV) [32] has a high computational complexity. Based on a large number of experimental tests, AR-MOEA uses IGD-NS as the main environmental selection strategy. IGD represents the average of the distance from each reference point in the Pareto fronts to the nearest solution, and IGD-NS adds a noncontributing elimination process based on IGD which means that the elimination does not affect the IGD index value. The advantage of IDG-NS is that the computational complexity is very low, and better solutions are easy to be recognized. The smaller the value of IGD-NS, the better the distribution of the population, and the closer to the Pareto fronts. The calculation formula of IDG-NS is

$$
I G D_{N S(P, R)}=\sum_{\substack{r \in R \\ p \in P}} \min \|p-r\|+\sum_{\substack{q \in Q \\ r \in R}} \min \|q-r\|,
$$

where $P$ is the population to be evaluated. $R$ is a set of reference points on the Pareto front surfaces. $Q$ is the set of all noncontributing solutions in the population $P$.

(2) Adaptive strategy of reference point

Since the Pareto fronts of the problem to be solved are unknown, the AR-MOEA will estimate the Pareto fronts by the shape of the current population in the objective space, and generates a set of uniformly distributed points on the simplex as the reference point set. Meanwhile, the reference point set is dynamically adjusted according to the contribution degree of the solution in the optimization process to ensure that the reference point set maximizes the irregular front surface of different shapes. The adaptive strategy mainly includes the following three steps:

Step 1: Normalized population $P$, external archive $A$, and initial reference point set $R$.

The point sets $\mathrm{a}$ and $\mathrm{b}$ of the minimum objective and the maximum objective of each dimension in the current population $P$ are selected, respectively. The normalization method is to subtract a from the target objective of the solution in $P$ and $A$, and multiply the objective value of the point in $R$ by $(b-a)$.

Step 2: Update external archive $A$.

First, delete all the repeated solutions and the governed solutions in $A$, and copy all the contributing solutions in $A$. Secondly, in order to increase the distribution of the solutions, adjust the position of the reference points of $R$ according to the position of the solution in $A$, do not change its distribution, and update $R$ to $R^{\prime}$. Finally, retain the contribution solution in $A$, if its scale is smaller than the scale of $R$, continue to select the least crowded solution in $A$, forming $A^{\prime}$.

Step 3: Update the adaptive reference point set $R^{\prime}$.

Identifying the reference point in the distance $R$ that has the closest contribution to the solution $A$, and keeping it as the effective reference point, and continuing to select a certain number of least crowded solutions in $A^{\prime}$, together with the effective reference point, to form an adaptive reference equal in size to $A^{\prime}$ point set $R^{\prime}$. In other words, $R^{\prime}$ is composed of the effective reference point in the initial reference point set $R$ and the partial solution in the external archive $A^{\prime}$, which means $R^{\prime}$ not only has the uniformity of the initial reference point set, but also can dynamically adjust the distribution of reference points based on the external document $A^{\prime}$.

(3) Environmental selection strategy

AR-MOEA shifts the minimum objective value of $P$ per dimension in $P$ to 0 , and performs non-dominated sorting. This method calculates the contribution of the solution in each non-dominated level at that level, and removes one solution that with the least contribution. A loop repeats the calculation until the union of the solutions of all the dominant levels equals $P$ and stops and enters the next generation loop. 


\subsection{Evaluation Index}

In this paper, four indicators of the calculation time, the number of Pareto solutions, the water supply guarantee rate, and the HV [32] were selected to evaluate the applicability of the multi-objective algorithm, among which

(1) In theory, the nondominated solution must be an optimal feasible solution, however, due to the constraint problem of the objective setting, the total amount of water transfer may appear greater than, equal to, or less than the planned situation. In the game of water shortage index, ecological water supply and net power generation, the more Pareto solutions that meet the total water demand, the more beneficial to the decision-making of engineering management. Therefore, this paper sets the water supply guarantee rate to calculate the ratio of the number of Pareto solutions that meet the total water transfer requirement to the total number of Pareto solutions. The larger the value, the better.

(2) As the theoretical Pareto fronts are still unknown, the calculation process of the index HV does not require the front surface to be known, and the higher the degree of recognition, the larger the value and the better the convergence and distribution of the Pareto solution. This paper normalizes the three objective values and calculates HV due to the different dimension.

(3) HV is defined as the super volume of the area surrounded by the points in the population $P$ and the points in the reference point set $R$. The larger the super volume, the better the convergence and distribution of $P$.

$$
\begin{gathered}
\operatorname{HV}(P, R)=\gamma(\operatorname{HV}(P, R)), \\
\operatorname{HV}(P, R)=\{\mathbf{z} \in \mathrm{Z} \mid \exists \mathbf{p} \in \mathrm{P}, \exists \mathbf{r} \ni \mathrm{R}: \mathbf{p}<\mathbf{z}<\mathbf{r}\}, \\
(\operatorname{HV}(P, R))=\int_{\mathrm{R}^{\mathrm{M}}} 1_{\mathrm{HV}(P, R)}(\mathbf{z}) \mathrm{dz} .
\end{gathered}
$$

\subsection{Parameter Set}

(1) The number of solving objectives of the four algorithms is 3, the water pumping flow of Huanginxia pumping station is the decision variable, the decision variable number is 672 , the population size is 2000 , the evolutionary algebra is 10,000 , and the crossover probability is 0.2 , the probability of variation is 0.3 , and the distribution index is 30 .

(2) The aggregation function in MOEA/D is the Tchebycheff function, the neighborhood range is 200, the neighborhood selection probability is 0.8 , and the maximum number of solutions in the child replacement population is 20 .

(3) The fitness scale factor in the IBEA is 0.08 .

(4) In the performance evaluation, the multi-objective scheduling model established in Section 3 is the fitness function, the number of simulation calculations is 30 . The maximum value, the minimum value, the average value, and the standard deviation were used to evaluate the performance of the algorithm.

\section{Results and Discussion}

\subsection{Algorithm Applicability Analysis}

Embed the multi-objectives optimization model into the PlatEMO platform [20] to optimize operation, and the evaluation indexes of the four algorithms are counted, respectively, according to the Pareto solution. The results are shown in Figure 3. 

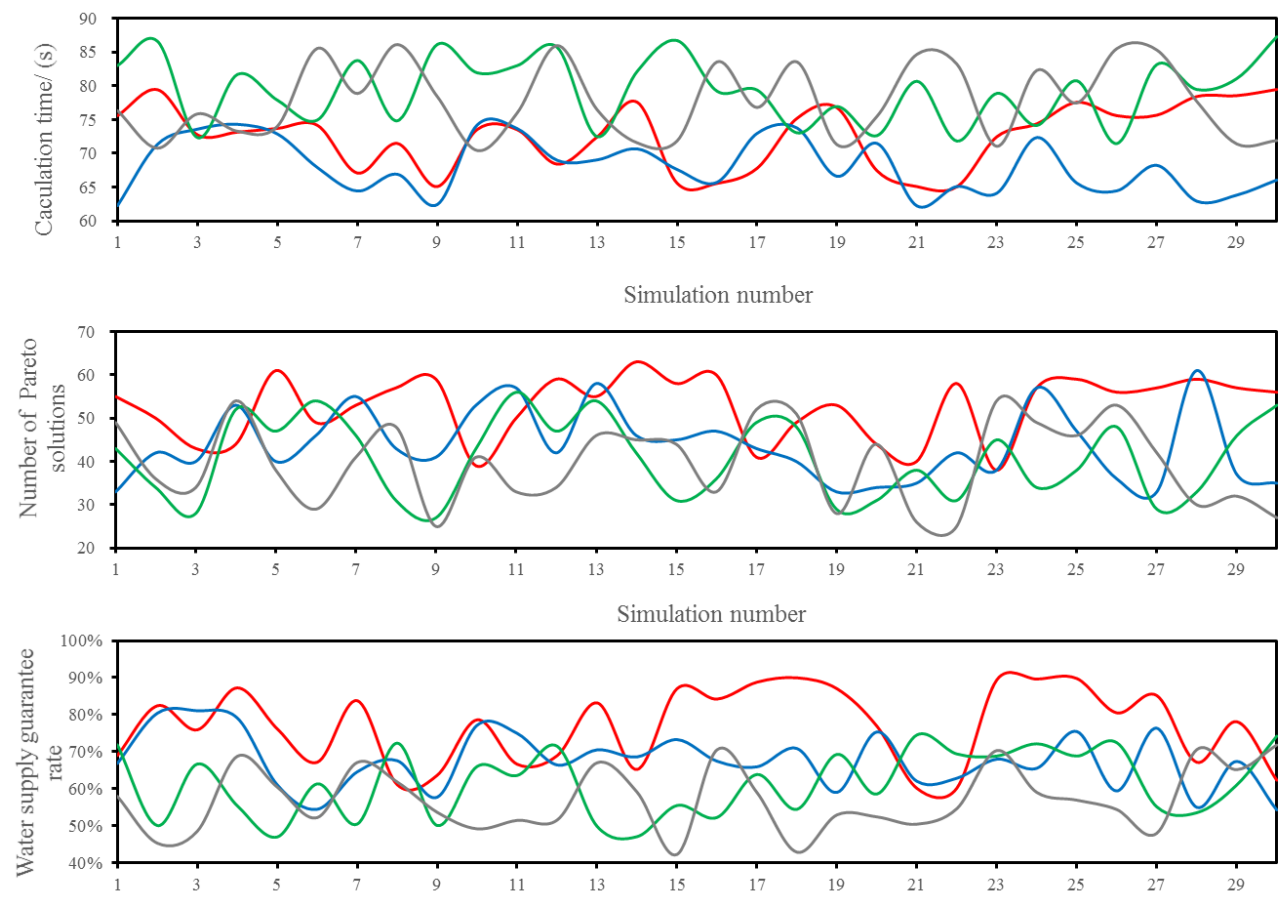

Simulation number

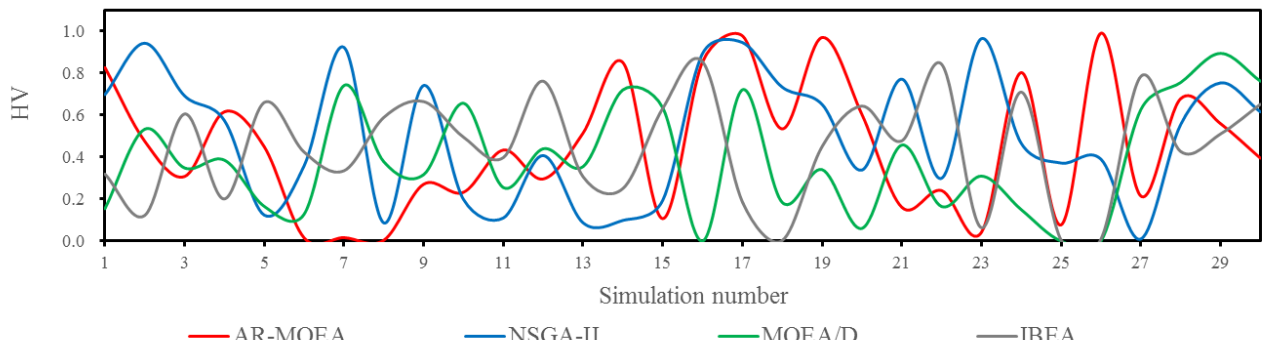

Figure 3. Evaluation index evaluation process of four kinds of algorithms.

As shown in Figure 3:

(1) Fluctuations: in the calculation process of 30 times, the results of the four algorithms all showed some fluctuations. Among them, the fluctuations of the calculation time, the number of Pareto solutions, and the water supply guarantee rate are relatively small, and the fluctuation of the $\mathrm{HV}$ is large. Because evolutionary algorithms are the randomness and initial populations are different, therefore, model is optimized by different starting points and directions.

(2) Evaluation index comparison: among the four algorithms, NSGA-II has the shortest calculation time, followed by AR-MOEA (red solid line), and other algorithms take longer to calculate. AR-MOEA is in a leading position in the number of Pareto solutions and the water supply guarantee, which means the conversion rate and optimization effect of non-dominated solutions in the whole population are relatively the best, followed by NSGA-II and MOEA/D. The HV indexes of the four algorithms vary greatly. The numbers of the HV value of 0 of the AR-MOEA, NSGA-II, MOEA/D, and IBEA were 2, 1, 3, and 2, respectively. It indicates that there is no convergence in this calculation. The reason is that the limit of the number of iterations causes the loop to end, but the overall convergence of the four algorithms is better.

In order to further evaluate the stability of the algorithm, the statistical values of the performance indexes of the four algorithms were calculated, which is shown in Table 2. 
Table 2. Statistical values of evaluation indexes of four algorithms.

\begin{tabular}{|c|c|c|c|c|c|c|c|c|}
\hline \multirow{2}{*}{ Algorithm } & \multicolumn{4}{|c|}{ Time (s) } & \multicolumn{4}{|c|}{ Number of Pareto Solutions } \\
\hline & $\begin{array}{l}\text { Maximum } \\
\text { value }\end{array}$ & $\begin{array}{l}\text { Minimum } \\
\text { value }\end{array}$ & $\begin{array}{c}\text { Average } \\
\text { value }\end{array}$ & $\begin{array}{l}\text { Standard } \\
\text { deviation }\end{array}$ & $\begin{array}{c}\text { Maximum } \\
\text { value }\end{array}$ & $\begin{array}{l}\text { Minimum } \\
\text { value }\end{array}$ & $\begin{array}{c}\text { Average } \\
\text { value }\end{array}$ & $\begin{array}{l}\text { Standard } \\
\text { deviation }\end{array}$ \\
\hline AR-MOEA & 79.47 & 65.09 & 72.62 & 4.37 & 63 & 42 & 52.63 & 7.40 \\
\hline NSGA-II & 74.31 & 62.25 & 68.20 & 4.03 & 61 & 33 & 43.73 & 8.38 \\
\hline MOEA/D & 87.25 & 71.48 & 79.43 & 4.97 & 56 & 27 & 40.77 & 9.10 \\
\hline IBEA & 86.11 & 70.44 & 77.76 & 5.40 & 54 & 25 & 39.63 & 9.45 \\
\hline \multirow{2}{*}{ Algorithm } & \multicolumn{4}{|c|}{ Water Supply Guarantee Rate } & \multicolumn{4}{|c|}{ HV } \\
\hline & $\begin{array}{l}\text { Maximum } \\
\text { value }\end{array}$ & $\begin{array}{l}\text { Minimum } \\
\text { value }\end{array}$ & $\begin{array}{c}\text { Average } \\
\text { value }\end{array}$ & $\begin{array}{l}\text { Standard } \\
\text { deviation }\end{array}$ & $\begin{array}{c}\text { Maximum } \\
\text { value }\end{array}$ & $\begin{array}{l}\text { Minimum } \\
\text { value }\end{array}$ & $\begin{array}{c}\text { Average } \\
\text { value }\end{array}$ & $\begin{array}{l}\text { Standard } \\
\text { deviation }\end{array}$ \\
\hline AR-MOEA & $89.95 \%$ & $60.11 \%$ & $76.84 \%$ & 0.10 & 0.99 & 0 & 0.52 & 0.31 \\
\hline NSGA-II & $81.07 \%$ & $54.27 \%$ & $67.62 \%$ & 0.08 & 0.96 & 0 & 0.45 & 0.30 \\
\hline MOEA/D & $74.54 \%$ & $46.98 \%$ & $61.57 \%$ & 0.09 & 0.89 & 0 & 0.39 & 0.27 \\
\hline IBEA & $71.82 \%$ & $42.26 \%$ & $57.17 \%$ & 0.09 & 0.85 & 0 & 0.40 & 0.28 \\
\hline
\end{tabular}

As shown in Table 2:

(1) NSGA-II has the smallest average calculation time and the smallest standard deviation, which indicates that the calculation stability is better, and AR-MOEA is second.

(2) Compared with 1000 populations, the number of Pareto solutions of the four algorithms is relatively small. Among them, AR-MOEA has the largest number of Pareto solutions, followed by IBEA, which indicates that the conversion effect of the AR-MOEA is best. The reason is that the nonlinearity and discontinuity of the reservoir operation model will increase the difficulty of obtaining non-dominated solutions.

(3) In the limited Pareto solution, AR-MOEA has the highest water supply guarantee rate, which indicates that in the case of optimizing the ecological objective and the net electricity objective, AR-MOEA obtains the most solutions with multi-year average water transfer equal to or greater than 1.5 billion $\mathrm{m}^{3}$, and the solution effect is better, followed by NSGA-II.

(4) The HV value of AR-MOEA is the largest among the four algorithms, which indicates that the convergence and distribution of the AR-MOEA solution are better, followed by NSGA-II.

Above all, the comprehensive performance (calculation accuracy, convergence, and distribution) of AR-MOEA is better than NSGA-II, MOEA/D and IBEA in the multi-objective optimization model of the Hanjiang to Wei River Water Diversion Project established in this paper.

\subsection{Multi-Objective Operation Rules}

The optimal values of the objectives are inconsistent by comparing and analyzing the Pareto results obtained after running the four algorithms 30 times, and the results of AR-MOEA and NSGA-II are better, MOEA/D and IBEA are second. Due to space limitations, this paper only shows the best Pareto point set for the single operation of each algorithm, that is, the water shortage index of the water receiving area is as small as possible, the minimum duration of the two rivers is as large as possible, and the net power generation is as large as possible. In order to display the distribution rule of Pareto point set better, we performed surface interpolation on discrete objective solutions, as shown in Figure 4. 

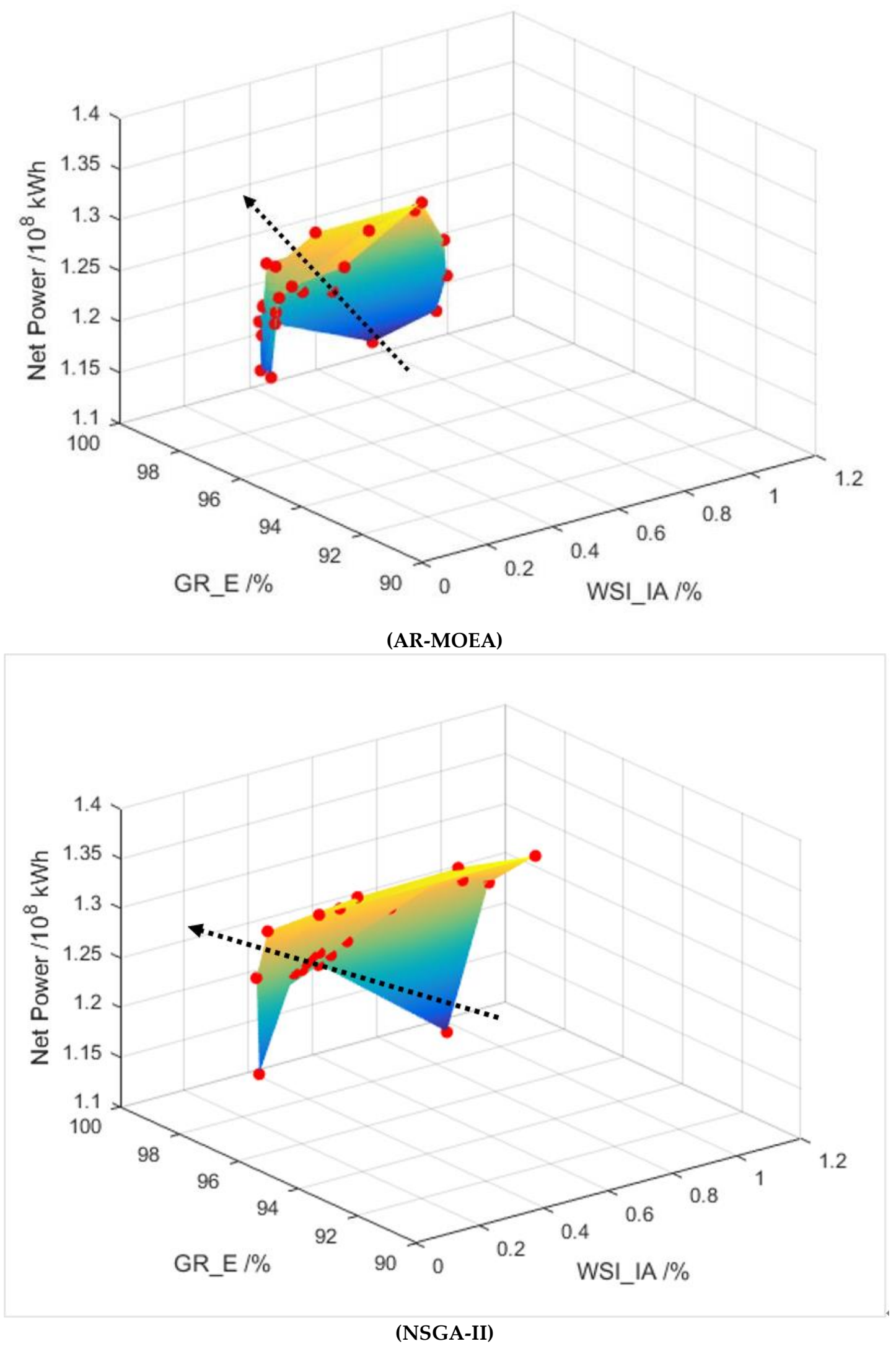

Figure 4. Cont. 

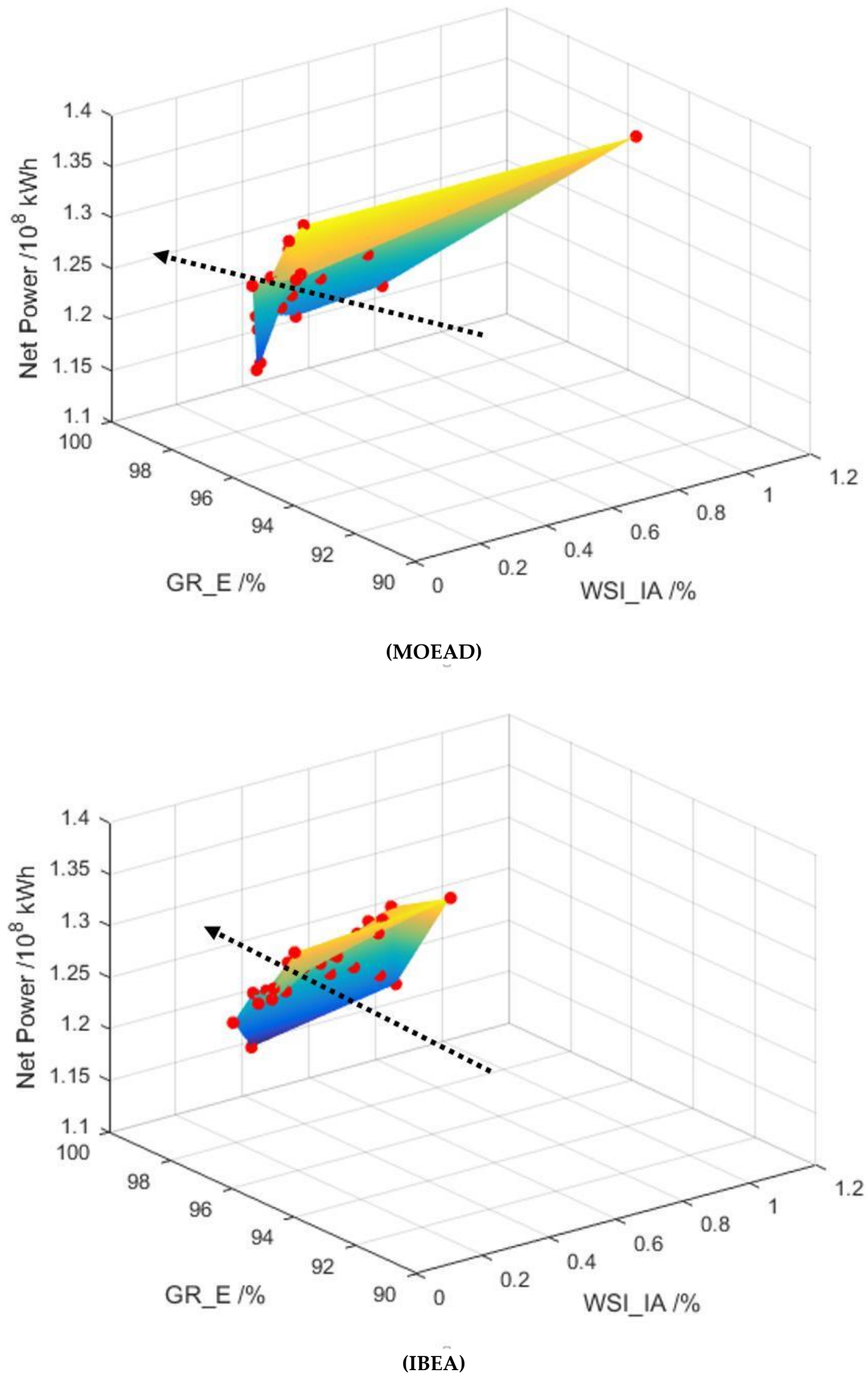

Figure 4. Pareto results of four algorithms. 
(1) Pareto extreme point.

As shown in Figure 3, there is a competing relationship between the three objectives. We counted the extreme values of the three objectives under the four algorithms together with the corresponding water transfer volume index. The optimal Pareto points of WSI_IA, GR_E, and P_net are shown in Table 3.

Table 3. Statistical values of four algorithms.

\begin{tabular}{|c|c|c|c|c|c|c|c|c|}
\hline \multirow[b]{2}{*}{ Algorithm } & \multicolumn{4}{|c|}{ AR-MOEA } & \multicolumn{4}{|c|}{ NSGA-II } \\
\hline & WSI_IA & $G R \_E$ & P_net & $\begin{array}{c}\text { Water } \\
\text { Transfer } \\
\left(10^{8} \mathrm{~m}^{3}\right)\end{array}$ & WSI_IA & $G R \_E$ & $P \_n e t$ & $\begin{array}{c}\text { Water } \\
\text { Transfer } \\
\left(10^{8} \mathrm{~m}^{3}\right)\end{array}$ \\
\hline WSI_IA (Min) & $0.008 \%$ & $94.799 \%$ & 1.294 & 15.010 & $0.000 \%$ & $95.394 \%$ & 1.293 & 15.014 \\
\hline$G R \_E$ (Max) & $0.429 \%$ & $96.285 \%$ & 1.193 & 14.931 & $0.662 \%$ & $96.137 \%$ & 1.170 & 14.902 \\
\hline P_net (Max) & $0.428 \%$ & $94.651 \%$ & 1.353 & 14.925 & $0.787 \%$ & $94.502 \%$ & 1.359 & 14.837 \\
\hline \multirow[b]{2}{*}{ Algorithm } & \multicolumn{4}{|c|}{ MOEA/D } & \multicolumn{4}{|c|}{ IBEA } \\
\hline & WSI_IA & $G R \_E$ & $P \_n e t$ & $\begin{array}{c}\text { Water } \\
\text { Transfer } \\
\left(10^{8} \mathrm{~m}^{3}\right)\end{array}$ & WSI_IA & $G R \_E$ & $P \_n e t$ & $\begin{array}{c}\text { Water } \\
\text { Transfer } \\
\left(10^{8} \mathrm{~m}^{3}\right)\end{array}$ \\
\hline WSI_IA (Min) & $0.004 \%$ & $95.394 \%$ & 1.296 & 15.012 & $0.002 \%$ & $95.988 \%$ & 1.261 & 15.013 \\
\hline$G R E$ (Max) & $0.204 \%$ & $96.137 \%$ & 1.238 & 14.963 & $0.518 \%$ & $96.285 \%$ & 1.249 & 14.933 \\
\hline P_net (Max) & $0.132 \%$ & $95.097 \%$ & 1.348 & 14.986 & $0.518 \%$ & $94.502 \%$ & 1.357 & 14.936 \\
\hline
\end{tabular}

As shown in Table 3, from the perspective of the WSI_IA value, NSGA-II is the best, followed by IBEA, MOEAD, and AR-MOEA, but the difference of the water transfer results of the four algorithms is very small. From the perspective of the GR_E value, AR-MOEA is close to IBEA, and NSGA-II is close to MOEAD. From the perspective of the $P \_n e t$ value, NSGA-II is the best, followed by IBEA, AR-MOEA, and MOEAD, but the MOEAD's water transfer is closer to 1.5 billion $\mathrm{m}^{3}$ obviously. Above all, from the perspective of optimizing the extreme objective, the performance of the four algorithms is close, especially in the existing strict constraints of the model, and the difference in Pareto extreme points is not significant.

(2) The evolutionary direction of the Pareto solution.

According to the setting of the optimization objective, this paper made a theoretical hypothesis: if we want to obtain the integrated Pareto solution of smaller WSI_IA, lager WSI_IA and lager $P \_n e t$, the interpolated surface of the entire Pareto point set should be convexly inclined into the Z-axis to obtain more solutions that match the three-way equalization effect. The evolution direction (red solid line in Figure 3) was formulated based on the position of the convex surface. As shown in Figure 3, the AR-MOEA is closer to the theoretical evolution direction of the hypothesis in all algorithms, and there are more Pareto solutions near the convex surface, followed by NSGA-II, MOEAD, and IBEA. Therefore, from the perspective of the Pareto solution evolutionary direction, the AR-MOEA has better performance.

(3) Pareto fronts.

It can be clearly seen from Figure 3 that (1) the shape of the Pareto surface solved by the four algorithms is upward convex, but the oblique direction of the convex tangent is inconsistent, which verified that although the objective values of some solutions are similar, the Pareto surfaces acquired by different algorithms are inconsistent, and it also depends on the actual modeling process and the situation of the constraints. (2) Compared with the other three algorithms, the Pareto point distribution of the AR-MOEA results is more uniform, and the corresponding surface is relatively smoother, which indicates that the AR-MOEA has better performance, and is more capable of searching and screening for non-dominated solutions when the Pareto front surfaces are unknown. 


\subsection{Coping with the Running Strategy of Continuous Dry Years}

It is can be obtained that Huangjinxiaand Sanhekou reservoir had a wet year during 1991-2002 by counting on the inflow runoff data. Among them, the Huangjinxia reservoir had four normal years, eight dry years, and the Sanhekou reservoir had five normal years and seven dry years. Both Huangjinxia and Sanhekou reservoir experienced dry years during 1991 and 2002. Especially, Sanhekou reservoir had two wet years after 2002. Therefore, we selected the continuous dry year sequence of the project from 1991 to 2002. In a single dry year, the storage capacity of the reservoir can be used to cope with the reduction of runoff. However, if there is a year with less water coming in for 12 consecutive years it will seriously affect the stability and guarantee rate of the water supply project, therefore, the study focused on the analysis of the operation of the project during a dry year period to determine the best water transfer rules.

According to the conclusions in Sections 5.1 and 5.2, the AR-MOEA can better solve the multi-objective optimization problem which the Pareto surface is unknown, especially the operation model solution of this project case. Therefore, a non-dominated solution was selected in the Pareto solution obtained by AR-MOEA calculation, and the corresponding water transfer process was analyzed. The water supply and water shortage of the Hanjiang to Wei River Water Diversion Project during 1991-2002 is shown in Figure 5, and the proportion of water supply in the Huangjinxia reservoir and Sanhekou reservoir is shown in Figure 6.

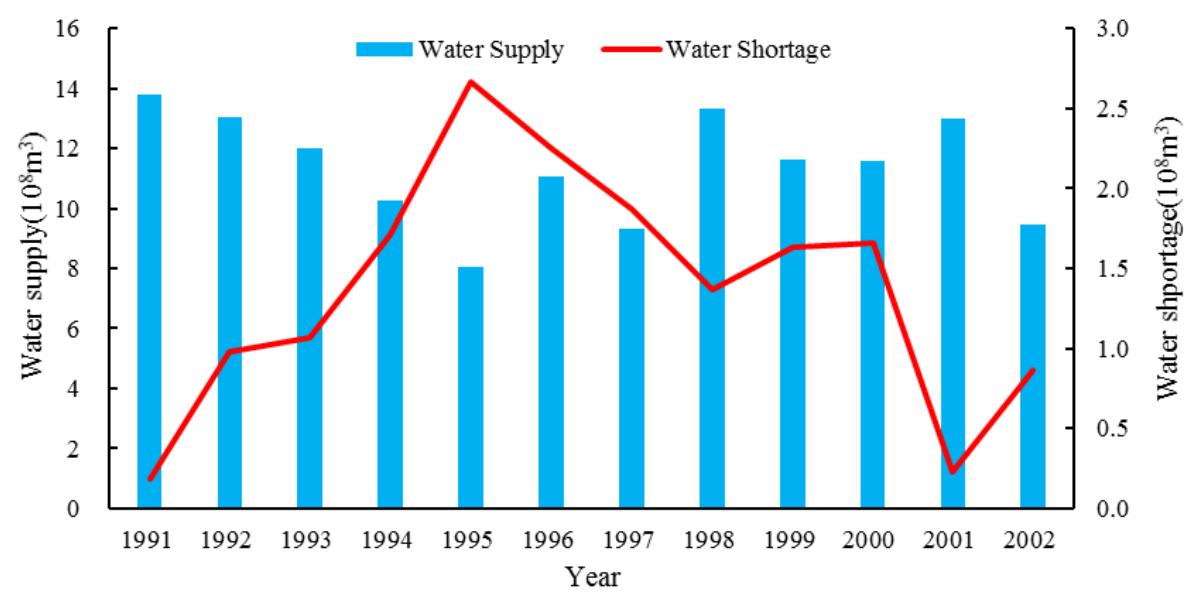

Figure 5. Water supply and water shortage of the Hanjiang to Wei River Water Diversion Project.

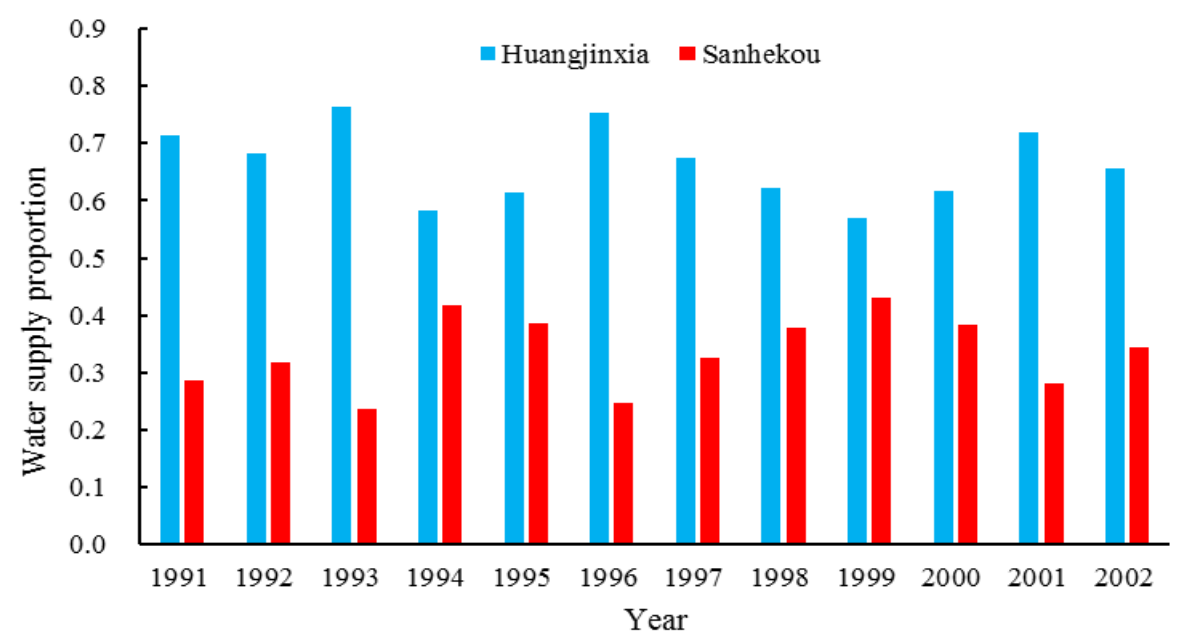

Figure 6. The proportion of water supply in the Huangjinxia reservoir and Sanhekou reservoir. 
As shown in Figures 5 and 6.

(1) Water shortages occurred in the continuous dry years of 1991-2002, which indicated that the stability and continuity of the water supply of the Hanjiang to Wei River Water Diversion Project were destroyed in this situation, and the maximum water shortage occurred in 1995, and the maximum water shortage was 267 million $\mathrm{m}^{3}$. The proportion of water supply indicates that the water supply of Huanginxia reservoir is the main source for the entire project compared to the water supply of Sanhekou reservoir.

(2) The water shortage showed a trend of increasing first and then decreasing during 1991-2002, the reason being that the storage water in Sanhekou reservoir can be used for water supply in the early stage of a continuous dry year, and as the dry time continues, the water shortage of the whole project is gradually exaggerated, but with the increase of runoff in the later period, the water shortage of the whole project is gradually reduced.

Furthermore, the monthly water supply flow process of the Huangjinxia reservoir and Sanhekou reservoir were calculated and are presented in Figure 7.

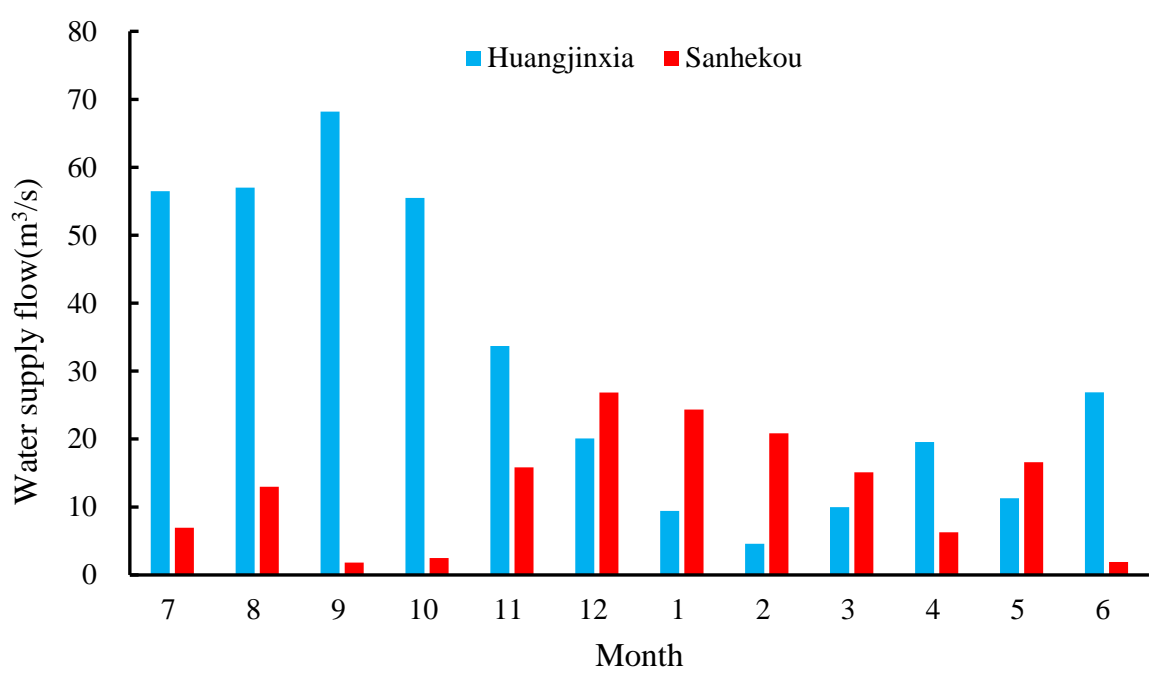

Figure 7. Monthly water supply flow process of the Huangjinxia reservoir and Sanhekou reservoir.

As shown in Figure 7.

(1) Overall, the water supply during the flood season (July-October) is much larger than the dry season (November-March (next year)) and the normal season (April-June). Further calculations show that the water supply in the flood season, dry season, and normal period accounted for $50 \%, 16 \%$, and $34 \%$ of the total water supply, respectively.

(2) The water supply of Huangjinxia reservoir and Sanhekou reservoir showed significant seasonality. The water supply of the Huangjinxia reservoir is significantly better than the Sanhekou reservoir from June to November, and the water supply of the Sanhekou reservoir is significantly larger than the Huangjinxia reservoir in December to March (next year). The reason is that the regulation ability of the Huangjinxia reservoir and the Sanhekou reservoir are daily regulation ability and multi-year regulation ability, respectively, which means that in the dry season, the former's water supply can only rely on natural runoff, while the latter can use its own regulation capacity to supply water.

In summary, in order to cope with the continuous dry years, the regulation ability of the Sanhekou reservoir should be fully exerted. In the case of the normal water supply of the Huangjinxia reservoir in the flood season, the storage capacity of the Sanhekou reservoir should be used as much as possible to store water, which can increase the water supply during the dry season, thereby increasing the water supply for the entire project in dry years. 


\section{Conclusions}

In this paper, a multi-objective model that considered water supply, ecology, and power generation was established and solved by four evolutionary algorithms, the performance of AR-MOEA was verified by using the Hanjiang to Wei River Water Diversion Project datasets.

(1) The applicability of AR-MOEA was compared to the other methods (NSGA-II, MOEA/D, and IBEA) for an example case from the Hanjiang to Wei River Water Diversion Project, and the results demonstrate that the AR-MOEA can achieve a better comprehensive performance in calculation accuracy, convergence, and distribution.

(2) Multi-objective operation rules based on the Pareto extreme point, the evolutionary direction of the Pareto solution, and the Pareto fronts indicate that the AR-MOEA has better performance and is more capable of searching and screening for non-dominated solutions when the Pareto front surface is unknown.

(3) The water supply in the flood season and the Huangjinxia reservoir are the main water supply factors of the Hanjiang to Wei River Water Diversion Project, but the regulation ability of the Sanhekou reservoir is the key factor to increase the water supply in response to the possible continuous dry years, especially during the dry season.

In addition to the above studies, this study lacks in-depth research on the sensitivity of parameters and reservoir scheduling rules in the calculation process. In future research, we should further explore the adaptability of the AR-MOEA, such as larger cascade reservoirs.

Author Contributions: Conceptualization, D.B.; methodology, J.X. and D.B.; Data curation, J.X.; investigation, D.B.; resources, D.B.; writing—original draft preparation, J.X.; writing—review and editing, J.X.; supervision, D.B.; project administration, D.B.; funding acquisition, D.B.

Funding: This study is supported by National Natural Science Foundation of China (41571222).

Acknowledgments: The authors are indebted to the reviewers and editors for their valuable comments and suggestions.

Conflicts of Interest: The authors declare no conflict of interest.

\section{References}

1. Fu, Z.H.; Zhao, H.J.; Wang, H.; Lu, W.T.; Wang, J.; Guo, H.C. Integrated planning for regional development planning and water resources management under uncertainty: A case study of Xining, China. J. Hydrol. 2017, 554, 623-634. [CrossRef]

2. Piao, S.; Ciais, P.; Huang, Y.; Shen, Z.; Peng, S.; Li, J. The impacts of climate change on water resources and agriculture in China. Nature 2010, 467, 43-51. [CrossRef] [PubMed]

3. Guo, Y.; Shen, Y. Quantifying water and energy budgets and the impacts of climatic and human factors in the haihe river basin, china: 2. trends and implications to water resources. J. Hydrol. 2015, 527, $251-261$. [CrossRef]

4. Guo, X.; Hu, T.; Zhang, T.; Lv, Y.B. Bilevel model for multi-reservoir operating policy in inter-basin water transfer-supply project. J. Hydrol. 2012, 424, 252-263. [CrossRef]

5. Li, Y.; Tang, C.; Wang, C. Assessing and modeling impacts of different inter-basin water transfer routes on Lake Taihu and the Yangtze River, China. Ecol. Eng. 2013, 60, 399-413. [CrossRef]

6. Jichuan, S.; Michael, W. Using incentives to coordinate responses to a system of payments for watershed services: The middle route of south-north water transfer project, china. Ecosyst. Serv. 2018, 32, 1-8.

7. Wu, L.; Bai, T.; Huang, Q.; Wei, J.; Liu, X. Multi-Objective Optimal Operations Based on Improved NSGA-II for Hanjiang to Wei River Water Diversion Project, China. Water 2019, 11, 1159. [CrossRef]

8. Zhou, Y.L.; Guo, S.L.; Hong, X.J.; Chang, F.J. Systematic impact assessment on inter-basin water transfer projects of the Hanjiang River Basin in China. J. Hydrol. 2017, 553, 584-595. [CrossRef]

9. Zenng, X.; Hu, T.S.; Guo, X.N.; Li, X.J. Triggering mechanism for inter-basin water transfer-supply in multi-reservoir system. J. Hydraul. Eng. 2017, 44, 253-261.

10. Guo, X.N.; Lei, X.H.; Li, Y.L.; Li, J.Q. Study on coupling of optimal water transfer and supply process for inter-basin water-transfer multi-reservoir system. J. Hydraul. Eng. 2016, 47, 949-958. 
11. Wan, F.; Zhou, J.; Yuan, W.L. Scheduling rules of multi-reservoir optimal operation in large-scale inter-basin water supply reservoirs. Adv. Water Sci. 2016, 27, 448-457.

12. Coello, A. An Updated Survey of Evolutionary Multiobjective Optimization Techniques: State of the Art and Future Trends. In Proceedings of the 1999 Congress on Evolutionary Computation-CEC99 (Cat. No. 99TH8406), Washington, DC, USA, 6-9 July 1999.

13. Reddy, M.J.; Nagesh, K.D. Multi-objective particle swarm optimization for generating optimal trade-offs in reservoir operation. Hydrol. Process. 2007, 21, 2897-2909. [CrossRef]

14. Yu, Y.; Wang, C.; Wang, P.; Hou, J.; Qian, J. Assessment of multi-objective reservoir operation in the middle and lower yangtze river based on a flow regime influenced by the three gorges project. Ecol. Inform. 2017, 38, 115-125. [CrossRef]

15. Uen, T.S.; Chang, F.J.; Zhou, Y.L.; Tsai, W.P. Exploring synergistic benefits of Water-Food-Energy Nexus through multi-objective reservoir optimization schemes. Sci. Total Environ. 2018, 633, 341-351. [CrossRef]

16. Niu, W.J.; Feng, Z.K.; Cheng, C.T.; Wu, X.Y. A parallel multi-objective particle swarm optimization for cascade hydropower reservoir operation in southwest China. Appl. Soft Comput. 2018, 70, 562-575. [CrossRef]

17. Qi, Y.; Bao, L.; Ma, X.; Miao, Q.; Li, X. Self-adaptive multi-objective evolutionary algorithm based on decomposition for large-scale problems: A case study on reservoir flood control operation. Inf. Sci. 2016, 367-368, 529-549. [CrossRef]

18. Bai, T.; Wei, J.; Chang, J.; Yang, W.; Huang, Q. Optimize multi-objective transformation rules of water-sediment regulation for cascade reservoirs in the Upper Yellow River of China. J. Hydrol. 2019, 577, 123-134. [CrossRef]

19. Ishibuchi, H.; Setoguchi, Y.; Masuda, H. Performance of Decomposition-Based Many-Objective Algorithms Strongly Depends on Pareto Front Shapes. IEEE Trans. Evol. Comput. 2017, 21, 169-190. [CrossRef]

20. Tian, Y.; Cheng, R.; Zhang, X. An Indicator-Based Multiobjective Evolutionary Algorithm with Reference Point Adaptation for Better Versatility. IEEE Trans. Evol. Comput. 2018, 22, 609-622. [CrossRef]

21. Zhou, Y.; Guo, S. Incorporating ecological requirement into multipurpose reservoir operating rule curves for adaptation to climate change. J. Hydrol. 2013, 498, 153-164. [CrossRef]

22. Zhou, Y.; Chang, L.C.; Uen, T.S.; Guo, S.; Xu, C.Y.; Chang, F.J. Prospect for small hydropower installation settled upon optimal water allocation: An action to stimulate synergies of water-food-energy nexus. Appl. Energy 2019, 238, 668-682. [CrossRef]

23. Deb, K.; Pratap, A.; Agarwal, S. A fast and elitist multiobjective genetic algorithm: NSGA-II. IEEE Trans. Evol. Comput. 2002, 6, 182-197. [CrossRef]

24. Arunkumar, R.; Jothiprakash, V. Optimal crop plans for a multi-reservoir system having intra-basin water transfer using multi-objective evolutionary algorithms coupled with chaos. Comput. Electron. Agric. 2017, 140, 34-47. [CrossRef]

25. Gadhvi, B.; Savsani, V.; Patel, V. Multi-objective optimization of vehicle passive suspension system using NSGA-II, SPEA2 and PESA-II. Procedia Technol. 2016, 23, 361-368. [CrossRef]

26. Zhou, Y.; Guo, S.; Chang, F.J.; Pan, L.; Chen, A.B. Methodology that improves water utilization and hydropower generation without increasing flood risk in mega cascade reservoirs. Energy 2018, 143, 85-796. [CrossRef]

27. Zhang, Q.; Hui, L. MOEA/D: A Multio-bjective Evolutionary Algorithm Based on Decomposition. IEEE Trans. Evol. Comput. 2007, 11, 712-731. [CrossRef]

28. Qi, Y.; Yu, J.; Li, X.; Wei, Y.; Miao, Q. Reservoir flood control operation using multi-objective evolutionary algorithm with decomposition and preferences. Appl. Soft Comput. 2017, 50, 21-33. [CrossRef]

29. Zhang, J.; Tang, Q.; Li, P.; Deng, D.; Chen, Y. A modified MOEA/D approach to the solution of multi-objective optimal power flow problem. Appl. Soft Comput. 2016, 7, 494-514. [CrossRef]

30. Zitzler, E.; Künzli, S. Indicator-Based Selection in Multi-Objective Search; Springer: Heidelberg/Berlin, Germany, 2004; pp. 832-842.

31. Strickler, A.; Castro, O.; Pozo, A.; Santana, R. An investigation of the selection strategies impact on moedas: Cma-es and umda. Appl. Soft Comput. 2017, 62, 963-973. [CrossRef]

32. Knowles, J.D.; Corne, D. Properties of an adaptive archiving algorithm for storing non-dominated vectors. IEEE Trans. Evol. Comput. 2003, 7, 100-116. [CrossRef]

(C) 2019 by the authors. Licensee MDPI, Basel, Switzerland. This article is an open access article distributed under the terms and conditions of the Creative Commons Attribution (CC BY) license (http://creativecommons.org/licenses/by/4.0/). 